\title{
Pediatric genitourinary oncology
}

\author{
Francisco Tibor Dénes ${ }^{1}{ }^{*}$, Ricardo Jordão Duarte ${ }^{1}$, Lílian Maria Cristófani ${ }^{2}$ and Roberto Iglesias Lopes ${ }^{1}$ \\ 1 Uropediatric Unit, Division of Urology, Hospital das Clínicas, University of São Paulo, São Paulo, Brazil \\ 2 Pediatric Onco-Hematology Unit, Department of Pediatrics, Hospital das Clínicas, University of São Paulo, São Paulo, Brazil
}

\section{Edited by:}

Raimund Stein, University of Mainz,

Germany

Reviewed by:

Nicholas Glenn Cost, Cincinnati

Children's Hospital Medical Center,

USA

Armando J. Lorenzo, Hospital for Sick

Children, Canada

Norbert Graf, Saarland University,

Germany

\section{*Correspondence:}

Francisco Tibor Dénes, Uropediatric

Unit, Division of Urology, Hospital das

Clínicas, University of São Paulo, Av.

Eneas Carvalho de Aguiar 255, São

Paulo 05403-000, Brazil

e-mail: ftdenes@gmail.com
Tumors of the kidney, bladder, prostate, testis, and adrenal represent a large part of the adult urologic practice, but are relatively infrequent in children. The natural history and management of these tumors in the pediatric age is different from that of the adults. As result of the successful work of several clinical trial groups in recent decades, there has been a significant improvement in their cure rates. The aim of this article is to review their most significant clinical aspects, as well as to present an update in their management.

Keywords: pediatric tumor, kidney neoplasms, rhabdomyosarcoma, testicular neoplasms, adrenal tumors

\section{KIDNEY TUMORS}

Among the primary pediatric kidney tumors, the most frequent is the Wilms tumor (WT), followed by the mesoblastic nephroma. The incidence of the different tumors is shown in Table $\mathbf{1}(1,2)$.

\section{WILMS TUMOR}

Wilms tumor or nephroblastoma is the most common genitourinary malignant tumor of children. The incidence in the United States is seven per million children below 15 years of age, while in different regions of Brazil it varies from 4 to 15 cases per million per year. The peak incidence occurs between 2 and 3 years of age $(3,4)$.

Mutations or deletions of the WT1 gene of the 11p13 locus are present in $15-20 \%$ of sporadic tumors, while the WTX gene shows mutations in $29 \%$ of them. Loss of heterozygosity ( $\mathrm{LOH}$ ) of the $1 \mathrm{p}$ and/or 16q chromosomes is a factor of adverse prognosis (5). Loss of imprinting (LOI) or LOH at $11 \mathrm{p} 15$ leads to IGF2 overexpression and is present in $70 \%$ of WT patients. Some syndromes and conditions are associated to WT gene mutations and to the presence of intralobar and perilobar nephrogenic rests, leading to an increased risk of developing WT, such as Beckwith-Wiedemann, Denys-Drash, WAGR, and corporal hemihypertrophy $(6,7)$.

The majority of WT is characterized by a triphasic histology, that includes blastematous, epithelial, and stromal components. The most important histological factor in prognosis is the occurrence of anaplasia, which is observed in $5 \%$ of the cases and is characterized by the presence of multiple mitoses, as well as increase in size and hyperchromasia of the cell nucleus. Its presence, either focal or diffuse, indicates an increased aggressiveness of the tumor, even if it is still localized, as in stages I and II (8).

In general, WT manifests itself as an asymptomatic abdominal mass, which is palpated by the parents or caretakers in $90 \%$ of the cases. Macroscopic hematuria occurs in $25 \%$ of cases. Abdominal pain is referred in $30 \%$, while high blood pressure is observed in about $25 \%$ of the patients. Up to $12 \%$ of patients with WT have associated congenital abnormalities, such as cryptorchidism, hypospadias, hemihypertrophy, and aniridia (9). Between 5 and $15 \%$ of patients have bilateral or multicentric tumors. The average age at presentation is $42-47$ months for patients with unilateral tumors and 30-33 months for those with bilateral tumors (7).

Usually, the abdominal ultrasound (US) examination confirms the presence of a renal mass, but computer tomography $(\mathrm{CT})$ is used to provide all information required for adequate diagnosis and staging of this tumor, including infiltration of adjacent structures, extension to the inferior vena cava or abdominal or thoracic metastases (7). In some cases, Doppler US or magnetic resonance imaging (MRI) may be necessary to confirm involvement of the renal veins and cava. Differential diagnosis includes neuroblastoma, which often occurs in children of the same age. The staging system for WT is presented in Table 2 (10).

Treatment of WT includes surgery, chemotherapy, and in some patients radiotherapy. There are two treatment protocols for the initial management of this tumor. One, recommended by the Children's Oncology Group (COG) advocates up-front surgical removal without interference of neoadjuvant antineoplastic drugs, thus providing a detailed histological staging and accurate cytogenetic studies, necessary for planning post-operative treatment. The other protocol is proposed by the International Society for Pediatric Oncology (SIOP), which advocates 4-6 weeks of chemotherapy before nephrectomy, with or without concomitant metastases. Despite the potential adverse effect of neoadjuvant chemotherapy in staging and histologic evaluation, its beneficial effects are the reduction of the tumoral size and the formation of a fibrous pseudocapsule that facilitate the surgical removal and decrease the risks of rupture and spillage during surgery. 
Table 1 | Pediatric kidney tumors: classification and frequency (1)

\begin{tabular}{lc}
\hline Histological type & Frequency (\%) \\
\hline Wilms' tumor & 85 \\
Mesoblastic nephroma & 5 \\
Clear cell sarcoma & 4 \\
Rhabdoid tumor & 2 \\
Miscellaneous & 4 \\
\hline
\end{tabular}

\section{Table 2 | Wilms tumor staging system (10).}

I - tumor restricted to the kidney, completely excised, no penetration of the capsule or involvement of renal sinus vessels

II - tumor beyond the kidney, but without residual beyond the margins of excision. Eventual tumoral thrombus outside the kidney, but completely removed en-bloc with the tumor

III - gross or microscopic residual local tumor postoperatively, including positive margins, inoperable tumor, intra-abdominal lymph node metastasis, peritoneal implants, tumoral rupture, or transected tumoral thrombus

IV - hematogenic metastases (liver, lung, bone, brain) or extra-abdominal lymph node metastasis

$\checkmark$ - bilateral tumor

In both protocols, chemotherapy is based on vincristine and dactinomycin for stage I and II with favorable histology and vincristine, dactinomycin, and doxorubicin for stage III and IV with favorable histology. Treatment of tumors with anaplastic histology, advanced stages (III and IV), or greater-risk tumors also includes cyclophosphamide or ifosfamide, carboplatin, and etoposide, eventually associated to radiation therapy (7). Stage V disease (bilateral tumors) requires preoperative chemotherapy with vincristine, dactinomycin, and eventually doxorubicin for 8 weeks, followed by nephron sparing surgery. In the long-term follow-up, the survival rates of both strategies, based in the surgical removal, pre and/or post-operative chemotherapy and radiotherapy, are equivalent $(10,11)$. The current trials of both protocols are trying to minimize the late effects of treatment without compromising the excellent overall survival (12).

The classical surgical procedure for the treatment of WT is open radical nephrectomy through a transperitoneal access $(10,13)$. The adrenal glands may be left "in situ," if they are unchanged and can be easily separated from the kidney; otherwise, they are removed. Although extensive lymphadenectomy is not required, perihilar and interaortocaval lymphnode samples must be obtained in all cases, as they are necessary to adequate staging and planning of post-operatory management. Failure to remove and evaluate lymphnodes increases the risk of local recurrence and significantly decreases the 5-year survival rate $(9,14)$.

According to the consolidated experience in the treatment of adult renal tumors, the well-known benefits of laparoscopic radical nephrectomy, namely reduced hospitalization and analgesic requirement, as well as the better cosmetic result, were extended to selected patients with WT. Although still considered experimental, this technique has been employed with good results in several centers in the treatment of unilateral tumors, with or without neoadjuvant chemotherapy, without impairing the oncological results (15-17).

Recent consideration has been given to nephron sparing surgery in selected patients with unilateral WT. While long-term, prospective data is not yet available, case series demonstrate preservation of renal function and comparable oncologic outcomes after partial vs. radical nephrectomy $(18,19)$.

In 5-7\% of the cases, WT involves both kidneys. In this scenario, besides complete removal of the tumor masses, treatment also aims preservation of as much healthy renal tissue as possible, by means of nephron sparing surgery (20). Preoperative tumor biopsy is not always required. Neoadjuvant chemotherapy is recommended for 6-12 weeks, after which the patient is re-evaluated by CT in order to plan the best surgical approach. Possible alternatives are bilateral partial nephrectomy or unilateral radical nephrectomy associated to contralateral partial nephrectomy. The risk of end-stage renal disease in bilateral WT is $5.4-12 \%$, compared to $0.2-0.6 \%$ in unilateral tumors (12). Bilateral radical nephrectomy is very rarely employed, as it implies immediate dialysis and later renal transplantation.

In $4-10 \%$ of cases, WT may present with tumoral thrombus in the renal vein, vena cava, or atrium, diagnosed by Doppler US, CT, or MRI $(10,13)$. If the tumor thrombus is above the hepatic veins, neoadjuvant chemotherapy is required to reduce the size of the tumor and of the thrombus. Those of the inferior vena cava may be extracted together with the tumor by incision of the vessel below the diaphragm. For those that reach above the diaphragm, thoracotomy, and cardiotomy with extracorporeal circulation is necessary.

Post-operative treatment depends on the stage of the disease, as well as the histological evaluation of the specimen and the lymphnodes obtained in surgery. As a rule, patients receive adjuvant chemotherapy in all stages, with the eventual exception of children younger than 2 years of age with stage I disease and tumors with favorable histology weighing $<550 \mathrm{~g}$. All stage III patients receive local irradiation, while in case of major tumoral rupture a whole abdominal radiotherapy is necessary (11). In the COG protocol, all patients with anaplastic histology also require radiotherapy $(10,11)$.

The 5-year event-free survival is estimated in $87 \%$ for stage I and $85-74 \%$ for stage II, $82 \%$ for stage III, and for stage IV it decreases to $60-70 \%$ (21). Recurrence in patients with stage I or II is associated to significant mortality even in favorable subtypes, with overall survival dropping to $50 \%$ despite the use of more intense chemotherapy in these relapsed cases (12).

Although significant success has been achieved in increasing the overall 5 -year survival rates to more than $90 \%$, multimodal therapy is associated to late adverse effects, which require longterm monitoring of these patients (12). Around $0.7 \%$ of patients with unilateral WT will develop renal failure, an incidence eightfold higher than that expected in the general population (22). Fifteen years after diagnosis, the cumulative incidence of a second malignancy is $1.6 \%$, with risk factors including abdominal radiotherapy as part of the initial or adjuvant treatment, with or without chemotherapy for relapse (23). In the NWTSG Late 
Effects Study, the cumulative incidence of congestive heart failure (CHF) 20 years after diagnosis of WT was $4 \%$ in patients whose treatment plan included doxorubicin, with a direct dose-response relationship (each $100 \mathrm{mg} / \mathrm{m}^{2}$ of doxorubicin exposure increased the relative risk of CHF by 3.3). Furthermore, patients treated for relapsed WT had a higher cumulative frequency of CHF compared with patients without relapse (24).

\section{MESOBLASTIC NEPHROMA}

Congenital mesoblastic nephroma $(\mathrm{CMN})$ is the most common solid kidney tumor of the newborns, also called renal hamartoma or leiomyomatous hamartoma. Usually, this tumor manifests itself in the first 3 months of life, with a slight predominance in males, as a painless abdominal mass. In some cases, it may be detected in the antenatal US, accompanied by polyhydramnios and hydropsy. It is also associated to early delivery.

At the time of detection, almost all CMNs are predominantly localized in the kidney and perinephric or hilar soft tissue. The imaging studies show a large, solid intrarenal mass that involves the sinus, generally presenting cystic, hemorrhagic, and necrotic areas (2). Histopathologically, they are predominantly monomorphic neoplasms composed of spindled mesenchymal cells of fibroblastic or myofibroblastic lineage. These are divided into two major types: classic and cellular. Cytogenetic evaluation of this tumor has shown that the cellular type is almost invariably associated with abnormalities of the $t(12 ; 15)$ and $11+$, whereas the classical type rarely is. These abnormalities are also observed in congenital fibrosarcoma (25).

Treatment is based on radical nephrectomy, and free margins are necessary due to the infiltrative tendency of this tumor. Prognosis is usually good, particularly in cases treated before the sixth month of life Recurrences and metastasis occur in approximately $5-10 \%$ of all tumors (26).

\section{RHABDOID TUMOR}

This is a rare tumor, with $80 \%$ of cases occurring in children before 2 years of age, mainly in boys, with a proportion to girls of 1.5:1 $(2,10)$. Some patients present synchronous brain lesions or atypical teratoid/rhabdoid tumor (AT/RT) that resemble primitive neuroectodermal tumors, and have the same genetic mutation (SMARCB1) found in the renal lesion (26). Approximately onethird of newly diagnosed patients with RT have an underlying genetic predisposition to tumors due to a germline SMARCB1 alteration. Family members may demonstrate incomplete penetrance and gonadal mosaicism, which must be considered when counseling parents of patients with RT (27).

The common symptom is hematuria, but often there are signs and symptoms related to metastatic spread to the lung, liver and brain, that are present at the time of diagnosis in up to $80 \%$ of the cases $(2,26)$. Preoperative biopsy is eventually required for pathological diagnosis, but early radical nephrectomy is necessary for cure. It is a very aggressive tumor, usually resistant to chemotherapy and radiotherapy. The 4-year survival rate varies from 20 to $36 \%(10,28)$.

\section{CLEAR CELL SARCOMA}

This tumor has a peak incidence between 1 and 4 years of age, with a predominance of boys over girls of $2: 1$. There are no known familiar or syndromic associations, nor bilateral cases $(2,16)$. The main sign is a palpable abdominal mass, and 15$60 \%$ of the cases present skeletal metastases at the time of diagnosis, with pain as the main symptom. The imaging studies do not allow differentiation from WT. This tumor is sensitive to chemotherapy, therefore it should be associated to radical nephrectomy as the treatment of choice. The 5-year relapse-free survival and overall survival rates reach as high as 79 and $86 \%$ respectively, for patients receiving 28-34 weeks of chemotherapy $(28,29)$.

\section{RENAL CELL CARCINOMA}

Renal cell carcinoma (RCC) is the diagnosis in $2-5 \%$ of the pediatric renal tumors. Between 0.5 and $2 \%$ of all RCC occur in patients younger than 21 years of age, mainly between 9 and 15 years $(2,10$, 26). It may be associated to the von Hippel-Lindau syndrome, in which the tumors are multiple and have an earlier occurrence (2). One population based study showed about $30 \%$ of cases associated to underlying disorders, like tuberous sclerosis, neuroblastoma, teratoma, Saethre-Chotzen syndrome, chronic renal failure, or related diseases in their family (30).

Clinical presentation is similar to that of adults, with painless macroscopic hematuria, flank pain, and palpable mass being the most frequent symptoms. In general, RCC is smaller than WT at the time of diagnosis. CT study shows a solid non-enhanced intrarenal lesion, with areas of hemorrhage, necrosis, and calcification, but clear differentiation from WT is not possible (2). At presentation, more than two thirds of the patients have localized disease. In metastatic disease, two thirds are in the regional lymph nodes and one-third in distant organs, mainly lung, liver, and brain $(2,30)$.

Childhood RCC differs histologically and biologically from those of adults. The papillary form occurs in $20-50 \%$ of the cases, while the rest is represented by the classical clear cell carcinoma $(10,26)$. Genetic translocations are found in $30 \%$ of the RCC in children, most of them involving the TFE3 gene on chromosome X p 11.2. Other biological targets of therapeutic interest may be present and include $c$-Met, $m T O R$, and VEGRF. Morphologically, these carcinomas resemble the conventional clear cell tumor, but present areas of papillary architecture. They represent a distinct class of renal carcinoma in the WHO classification (2, 10). Treatment consists in the complete removal of the tumor by radical nephrectomy, either by open or laparoscopic access. Lymphnode sampling is recommended for adequate staging. The adrenal gland may be preserved in cases of lower pole tumor. Initial experience suggests that equivalent cure rates can be expected from a nephron sparing approach in appropriately selected cases (31). This tumor is resistant to chemotherapy or radiotherapy, and the treatment of metastases is still a challenge. Angiogenesisinhibiting or immune-stimulating agents, like vaccines, interferon, sorafenib, sunitinib, and sirolimus, are possible therapeutic alternatives, with limited temporary results. Event-free survival and overall survival rates at 5 years reach $96 \%$ for localized RCC, 69 and $75 \%$ for regional lymph node-positive patients, 25 and $33 \%$ for patients with distant metastases, respectively (30). Recent studies show that survival in children with lymphatic spread only is better than in adults $(10,28)$. 


\section{MEDULLARY CARCINOMA}

Described recently in 1995, it affects carriers of sickle-cell disease trait. It presents a very aggressive behavior, with frequent metastases and a mortality rate close to $100 \%$. Treatment is based on radical nephrectomy, as it is unresponsive to chemo or radiotherapy (28).

Other less common renal malignancies of childhood include the anaplastic sarcoma, cystic nephroma, primitive neuroectodermal tumor, desmoplastic small round cell tumor, as well as the intrarenal neuroblastoma. Adenomas, lipomas, lymphomas, and angiomyolipomas are also described in children. In these, as in the previously described tumors the preoperative imaging studies are mostly unable to establish the preoperative diagnosis. Their treatment is mostly based on radical nephrectomy (12).

\section{RHABDOMYOSARCOMA}

Rhabdomyosarcoma (RMS) is a malignant tumor that originates from the embryonic mesenchymal cells that give origin to the striated musculature. It may surge in various sites in the body, even where the striated muscle is not usually present. They represent $4-8 \%$ of the malignant tumors in patients younger than 15 years of age, and around $21 \%$ of them rise in the genitourinary tract, mainly in the bladder, prostate, vagina, and the paratesticular region (32).

The annual incidence in the United States is 4.5 cases per million children younger than 15 years of age. There is a bimodal age distribution with a peak incidence in the first 2 years of life and another in adolescence. Etiology is unknown, but a possible explanation is the regulatory disruption of striated muscle progenitor cell growth and differentiation (33). Some environmental factors are associated to an increased risk for developing the disease, like paternal smoking habit, advanced maternal age, intrauterine exposition to radiation, and use of illicit drugs by the mother. A genetic predisposition is found in the Li-Fraumeni syndrome and neurofibromatosis. Compared to the general population, patients with RMS have an increased incidence of associated congenital malformations (32 vs. 3\%) $(34,35)$.

Rhabdomyosarcoma includes a heterogeneous group of tumors, that present two main histologic variants: the embryonal, which is predominant, and the alveolar. The embryonal RMS is subdivided into spindle cell and the botryoid sarcoma subtypes. Spindle cell histology is common in paratesticular lesions, whereas botryoid lesions are polypoid masses that fill the lumen of the bladder and vagina $(33,35)$. Embryonic RMS generally presents a favorable prognosis. Alveolar histology, which is characterized by two genetic reciprocal translocations PAX3-FOXO1 and PAX7$F O X O 1$, is more prevalent in adolescents and confers a lesser chance of cure due to the high rate of local recurrence $(32,34,36)$.

The clinical symptoms of genitourinary RMS depend on the site of the lesion. Paratesticular RMS presents as unilateral painless scrotal mass distinct from the testis, which is fortuitously palpated. At diagnosis, retroperitoneal lymph node extension is detected in $20-40 \%$ of these patients. Bladder or prostatic tumors generally cause voiding symptoms like frequency, dysuria and hematuria, but urinary retention is the most frequent. On physical examination, a pelvic mass can be palpated. Girls with primary tumor in the vagina or uterine cervix may present in the first years of life with vaginal bleeding or discharge and occasional exteriorization of the polypoid mass through the vulva $(34,35)$.

Patients with suspected bladder, prostate, and vaginal tumors require complete laboratorial and imaging studies. MRI provides excellent definition of the primary tumor and surrounding tissues, while CT is useful for evaluation of bone erosion and abdominal lymphadenopathy. Bone scan is necessary for the search of skeletal metastases, while the PET-scan is useful in the evaluation of the primary lesion as well as regional and distant metastases $(33,37)$. Diagnosis is confirmed by biopsy or resection of the tumor.

Paratesticular tumors are best managed initially by radical orchiectomy through the inguinal approach.

According to the recommendations of the Intergroup RMS Study Group, most boys older than 10 years of age should then undergo staging ipsilateral retroperitoneal template lymph node dissection followed by multiagent chemotherapy and radiotherapy. In children aged $<10$ years, however, PET-CT may be sensitive for the identification of metastatic retroperitoneal lymphatic metastases, thus reducing the need of lymphadenectomy (38). Radiotherapy is beneficial in patients with lymph node-positive disease (39).

Bladder or prostatic lesions require cystoscopic evaluation and biopsy, but when these are unsuccessful, needle biopsy or open surgery may be necessary. Protruding vaginal masses are easily biopsied. All samples must be submitted to histopathological, immunohistochemical, cytogenetic, and molecular studies. Presently, the main therapeutic objective in vesico-prostatic or vaginal-uterine RMS is to ensure cure with maximal functional and anatomical preservation of the involved organs, avoiding mutilating procedures that are associated to increased morbidity. After imaging and pathological studies, neoadjuvant chemotherapy is initiated. With size reduction of the tumor, definitive but conservative surgical resection is recommended. The role of surgery is decisive in the success of the treatment, since local control of the disease correlates with prognosis. Complete resection of the primary tumor is ideal. Microscopic evaluation of the surgical margins, as well as lymphnode sampling for staging, is highly recommended. Adjuvant chemo and radiotherapy are then initiated according to the stage of the disease and the success of surgery (40).

Rhabdomyosarcoma is staged by the Soft Tissue Sarcoma Committee with a disease-specific TNM staging system (Tables 3 and 4) (41). Considering the stage, group and histology, patients are assigned to low, intermediate, or high-risk groups. Those with paratesticular and vaginal RMS with embryonic histology and complete resection, even with microscopic residual, are considered low-risk patients. Intermediate-risk patients include bladder and prostatic RMS, as well as those with gross residual disease. High-risk patients are those with metastatic disease (40-42).

Patients with positive lymph nodes, also when detected by PET-CT, must be submitted to more intensive chemotherapy and eventual radiotherapy. This multimodal approach ensures survival rates of $90 \%(35,43)$. In tumors that involve the bladder and prostate, around $70 \%$ are originated in the bladder. In most of these cases, neoadjuvant chemotherapy and radiation allow a bladder sparing surgery. It is possible to achieve disease-free survival with preservation of vesical function by partial cystectomy in $50-60 \%$ of cases, particularly in those with tumors arising in the 
Table 3 | Preoperative staging of rhabdomyosarcoma (41).

\begin{tabular}{llllll}
\hline Stage & Organ & Tumor (T) & $\begin{array}{c}\text { Size } \\
\text { Lymphnode } \\
\text { (N) }\end{array}$ & $\begin{array}{l}\text { Metastases } \\
\text { (M) }\end{array}$ \\
\hline I & $\begin{array}{l}\text { Paratesticular, } \\
\text { vaginal uterine }\end{array}$ & T1 orT2 & a, b & N0, N1, Nx & M0 \\
II & Bladder, prostate & T1 orT2 & a & N0, Nx & M0 \\
III & Bladder, prostate & T1 orT2 & a & N1 & M0 \\
& b & N0, N1, Nx & & & \\
IV & All & T1 orT2 & a, b & N0 or N1 & M1
\end{tabular}

T1, tumor confined to site of origin ( $a$, diameter $<5 \mathrm{~cm} ; b$, diameter $>5 \mathrm{~cm}$ ).

$T 2$, local infiltration, extension, or adherence $(a$, diameter $<5 \mathrm{~cm}$; $b$, diameter $>5 \mathrm{~cm})$.

No, negative regional lymph nodes; N1, positive regional lymph nodes. Nx, lymph nodes status unknown; Mo, no distant metastases; M1, positive distant metastases.

Table 4 | Post-operative classification of rhabdomyosarcoma (41).

\begin{tabular}{cl}
\hline Group 1 & $\begin{array}{l}\text { Localized disease, completely removed, without } \\
\text { microscopic residual }\end{array}$ \\
B & Confined to the site of origin, but completely removed \\
Infiltration beyond site of origin, but completely removed \\
A & Total macroscopic resection \\
& Macroscopic resection with evidence of microscopic \\
B & Regidual lesion \\
C & resected without microscopic residual lesion \\
& Local microscopic residual lesion and/or residual lymph \\
Group 3 & Incomplete resection or biopsy, with residual mass \\
Group 4 & Distant metastases \\
\hline
\end{tabular}

bladder dome. Larger tumors, as well as those originating from the bladder base or prostate and also those resistant to neoadjuvant chemotherapy eventually require extensive resection with ureteral reimplantation, cystectomy, or cystoprostatectomy, with need of urinary diversion (44). In general, tumors that originate from the vagina respond well to chemotherapy, rarely requiring procedures like partial vaginectomy or vaginectomy with hysterectomy. Late complications like vaginal stenosis, ureteral obstruction, intestinal stricture or fistula, and ovarian failure are often observed after multimodal therapy (42).

Chemotherapy is based in the use of vincristine, actinomycin $\mathrm{D}$, and cyclophosphamide for low-risk patients. The group of intermediate-risk may receive the same drugs associated to ifosfamide or etoposide. High-risk patients must be treated with a combination of ifosfamide, etoposide, and doxorubicin. The combination of vincristine and irinotecan is useful in metastatic disease. The aim-therapy for $m$ TOR receptors, employing rapamycin, temsirolimus, and everolimus, as well as IGF-1R, is still under investigation. Children with low-risk tumors have 5-year survival rates of $90 \%$, while those with intermediate-risk reach $55-70 \%$ and those with high-risk <50\% (41-43, 45).

\section{TRANSITIONAL CELL CARCINOMA}

Transitional cell carcinoma of the bladder in children is very rare, with about 150 patients younger than 20 years presented in the literature, only $20 \%$ of them in the first 10 years of age. It predominates in white children with a male-to-female ratio of 3:1 (46-48).

Asymptomatic macroscopic hematuria is the most common onset symptom. Imaging studies with US followed by cystoscopy are the ideal diagnostic tools. There are few isolated reports of upper urinary tract involvement, therefore the kidneys must always be evaluated by ultrasound. Most lesions are located in the trigone, and the predominant histological pattern is papillary and noninvasive. Endoscopic resection is the standard treatment, being effective in all cases of localized disease. Follow-up includes regular ultrasound evaluation and urinary cytology every 6 months in the first 2 years and cystoscopy once a year. Recurrence is described in $13 \%$ of the patients, and progression to invasive disease with muscular involvement occurs in $10-15 \%$ of cases $(48,49)$.

\section{TESTICULAR TUMORS}

Testicular tumors are less frequent in childhood than in postpubertal age. The annual incidence is $5.9 / 100,000$ boys younger than 15 years of age, with the peak incidence in the first 3 years of life. They represent $2 \%$ of the pediatric tumors, and two thirds of them are of benign nature (Table 5$)(50,51)$.

Increase in the scrotal volume and palpation of a hardened painless testicular mass are the initial findings in $88 \%$ of the patients. In some cases, a hydrocele or hernia may lead to the diagnosis. Inflammatory processes, as well as cysts and particularly testicular torsion must be excluded (53). Physical examination is important, in order to evaluate signs of virilization. The preferred imaging modality for these tumors is doppler US, that identifies the presence, size, and exact location of the mass, but does not differentiate between benign and malignant lesions. Nevertheless, some tumors have specific US characteristics, like the epidermoid cysts and the teratoma. Alpha-feto-protein (AFP) is an important tumor marker, and has increased blood levels in most cases of malignant prepuberal testicular tumors. Human chorionic gonadotropin ( $\beta$-HCG) blood levels are rarely increased in these tumors. In confirmed malignant tumors, it is necessary to search for retroperitoneal and pulmonary metastases with CT imaging. Staging is based in image studies and tumoral markers, as well as the pathological findings (Table 6).

Surgical treatment usually begins with radical orchiectomy, which is recommended whenever the AFP is elevated. It is performed through an inguinal incision with control of the vessels at the internal inguinal ring and removal of the testis without violating the vaginal tunic. If the AFP is normal, the tumor is probably benign, as in cases of teratoma and epidermoid cyst. A partial orchiectomy may be then considered, requiring protection of the operative field, clamping of the pedicle, incision of the vaginal tunic and albuginea, and careful enucleation of the mass. The tunics are then closed, and the testis reinserted in the scrotum after clamp removal $(52,54)$. 
Table 5 | Relative frequency of the different tumor types registered in the "prepuberal testis tumor registry" (52).

\begin{tabular}{lrrl}
\hline Type of tumor & N & \% & Behavior \\
\hline Yolk sack tumor & 244 & 62 & Malignant \\
Teratoma & 92 & 23 & Benign \\
Indifferentiated stromal & 16 & 4 & Occasionally malignant \\
Epidermoid cyst & 13 & 3 & Benign \\
Juvenile cell of granulosa & 11 & 3 & Benign \\
Sertoli cell & 10 & 3 & Malignant in older children \\
Leydig cell & 5 & 1 & Benign \\
Gonadoblastoma & 4 & 1 & Usually benign \\
& & & \\
\hline
\end{tabular}

Table 6 | Children's Oncology Group (COG) staging system for malignant testicular tumors of childhood (53).

\begin{tabular}{ll}
\hline Stage & Extent of disease \\
\hline I & $\begin{array}{l}\text { Limited to testis, completely resected by high inguinal } \\
\text { orchiectomy. Absence of clinical, radiographic, or histologic } \\
\text { evidence of residual disease }\end{array}$ \\
& Transcrotal biopsy, microscopic disease in scrotum or in \\
& spermatic cord ( $<5 \mathrm{~cm}$ from proximal end). Tumor markers \\
& fail to normalize or decrease with appropriate half-life \\
& Retroperitoneal lymph node involvement, but no visceral or \\
& extra-abdominal involvement. Lymph nodes $>4 \mathrm{~cm}$ by CT, or \\
& $>2 \mathrm{~cm}$ and $<4 \mathrm{~cm}$ with biopsy proof \\
& Distant metastases, including liver
\end{tabular}

In the post-operative period, the patient is observed in case of benign tumors. In case of malignancy with stage I testes tumors, surgery alone presents excellent survival rates, with recurrence in $15.5 \%$ of the patients. Stage II disease is associated with $75 \%$ recurrence rate. In both cases, recurrence is successfully treated with chemotherapy based on cisplatin, etoposide, and bleomycin. Higher stages of the disease have also excellent prognosis, with association of surgery and chemotherapy (52).

\section{YOLK SACK TUMOR}

It is the most common testis tumor in children younger than 2 years of age, presenting as a solid testicular mass associated to increased levels of AFP (51). Most cases present with localized disease, and only $4-6 \%$ have retroperitoneal or pulmonary metastases, which are suspected by persistently increased levels of AFP after orchiectomy and evidenced by imaging studies.

Radical orchiectomy is generally curative in localized disease, therefore if the post-operative blood levels of AFP, whose half-life 5-7 days, are normal and the imaging studies are normal, there is no need of adjuvant therapy (55). Cases with persistently elevated APF or confirmed metastases must be treated with chemotherapy, presenting 6-year survival rates near 100\% (55-57).

\section{TERATOMA}

It is the second most frequent prepuberal testicular tumor, with a peak incidence at 13 months of age (55). Contrary to those that occur in the adult age, prepuberal teratomas are usually benign (58). Although not specific, the US finding of cysts within the tumor may suggest the diagnosis. As mentioned before, conservative surgical treatment is recommended in most cases, with careful resection of the nodule and preservation of the normal testicular tissue. However, in patients with signs of virilization, radical orchiectomy is indicated (59).

\section{EPIDERMOID CYST}

These lesions are composed entirely of keratin producing epithelium, which is easily identified in most cases by US evaluation, due to the peculiar aspect of concentric layers similar to that of an onion (60). This tumor probably represents a monodermal teratoma. It is usually benign and is best treated with conservative surgery $(50,51,54)$.

\section{STROMAL TUMORS}

These are very rare tumors in this age group. They are represented by the following four lineages (61):

- Leydig cell tumor: usually benign, it occurs between 5 and 10 years of age and can manifest with virilization. It may be treated with partial orchiectomy.

- Sertoli cell tumor: occurs between 4 months and 10 years of age. Although hormonally inactive in most cases, it can occasionally cause gynecomasty or early puberty. There are no reports of metastases in this age group, but older boys can present disseminated disease. Accordingly, simple orchiectomy may be curative in younger patients, but in older boys imaging studies are necessary to identify metastatic disease.

- Tumor of the juvenile cell of the granulosa: it is named by the presence of cells that resemble the granulosa layer of the juvenile ovary. Occurs mainly in the first semester of life and is associated to abnormalities of the $\mathrm{Y}$ chromosome. There may be an association with genital ambiguity. The usual treatment is simple orchiectomy.

- Undifferentiated stromal tumor: it presents areas of stromal neoplasia associated to undifferentiated areas of fusiform cells with high degree of mitoses. Most cases are benign, but in older boys they may present malignant behavior. Routine treatment is orchiectomy, but adjuvant chemotherapy may be necessary in cases of confirmed malignancy.

\section{GONADOBLASTOMA}

Patients with disorders of sexual differentiation present an increased incidence of gonadal tumors in the presence of the $\mathrm{Y}$ chromosome, particularly when associated to intra-abdominal cryptorchism or disgenetic gonads. Although more frequent in the post-puberal age, they may occur in childhood. They are usually benign and asymptomatic, but may be associated to virilization. If not removed, they may progress to disgerminomas, that present malignant behavior. Prophylactic laparoscopic gonadectomy is recommended in these cases $(61,62)$.

\section{ADRENAL TUMORS}

The adrenal gland may be site of several types of tumors, either benign or malignant, primary, or metastatic. Primary adrenal 
tumors originate from the medulla or cortex, and can be hyper or non-functioning. The group of primary medullary neoplasms include the neuroblastoma, ganglioneuroblastoma, and ganglioneuroma, which are collectively referred as neuroblastic tumors, as well as the pheochromocytoma (63). Tumors originating from the cortex include the adrenocortical adenoma and carcinoma. In children, the most common lesions are neuroblastomas and cortical adenomas, with pheochromocytomas and cortical carcinomas occurring more rarely (64).

Diagnosis of adrenal tumors is suspected in children with hormonal abnormalities. Routine US imaging may disclose nonfunctioning tumors in the minority of patients. Clinical evaluation must include imaging and hormonal studies. Abdominal CT or MRI confirm the side, size, and extension of the tumor, as well as the eventual presence of vascular thrombus, infiltration of adjacent structures and distant metastases.

\section{NEUROBLASTIC TUMORS}

The adrenal is the most common site of tumors originated from the ganglionar cells. These tumors include the neuroblastoma, which is the most malignant, due to the presence of undifferentiated small round cells, the ganglioneuroma, that is a more mature neoplasm, with a benign behavior, and the ganglioneuroblastoma, which presents mixed histology and has an intermediate behavior. Both neuroblastoma and ganglioneuroblastoma occur more frequently in children, while the ganglioneuroma presents more often in adolescents and young adults (63).

Neuroblastoma is the most common extracranial solid neoplasm in children, arising in the adrenal or in any segment of the sympathetic plexus. In $75 \%$ of the cases it is found in the abdomen, being one-third in the medulla of the adrenal gland. It is the most frequent malignant adrenal tumor in childhood, occurring more often during the first 10 years of life, $80 \%$ of them in children younger than 5 years of age. It may be diagnosed antenatally or in the first months of life on routine US examinations, but is most often detected as a palpable abdominal mass. Symptoms are related to the mass effect, with pain and abdominal distension, invasion of adjacent organs, metastatic disease or abnormal hormonal production of catecholamines, or vasoactive intestinal polypeptides. Opsomyoclonus or cerebellar ataxia has been observed in up to $4 \%$ of patients (63). The tumor consists mainly of primitive neuroblasts, and has a tendency to infiltrate and sometimes invade the adjacent organs and vessels, as well as to present hemorrhage and necrosis. Imaging studies with CT or MRI are necessary to stage the local extension of the lesion, while ${ }^{123}$ I-meta-iodobenzylguanidine scintigraphy is useful to identify distant metastases, mainly to the lymph nodes, bone marrow, liver, and skin, which are present in more than half of the patients at the time of diagnosis (63). Bone metastases are frequently detected in skull, vertebrae, and long bones (63). The prognosis for adrenal neuroblastoma depends mainly on the age of the patient and stage of the disease. In very small children and infants these tumors may spontaneously regress and even disappear (65). Except in this very early age group, in which conservative management may be employed, adrenalectomy is the procedure of choice, either by open or laparoscopic approach. The prognosis is dismal for children older than 18 months of age, with metastatic disease. Tumors presenting amplification of the MYCN oncogene have a bad prognosis and are considered as high-risk group (65). For these patients surgery with chemoradiotherapy, stem cell transplantation, as well as anti-disialoganglioside (GD2) immunotherapy plus cytokines may improve survival (66).

Ganglioneuroblastomas and ganglioneuromas occur more rarely, the former with similar symptoms as the neuroblastoma, the later more often asymptomatically. While differential diagnosis is difficult by imaging studies, urinary catecolamine levels are usually not increased in these tumors (63). Surgical removal is the standard treatment.

\section{PHEOCHROMOCYTOMA}

This neoplasm occurs very rarely in children and represents $<1 \%$ of all tumors in this age group. It usually occurs in older children, at a mean age of 11 years. Compared to adults, there is an increased tendency of bilaterality, rarely being malignant. Most of these lesions occur sporadically, but a familial heritage is possible, particularly in syndromes like Multiple Endocrine Neoplasia (MEN) type-2 and diseases like von Recklinghausen, von Hippel-Lindau, and Sturge-Weber (63).

Pheochromocytoma generally manifests itself by the increase in blood pressure levels, sweating, tachycardia, headaches, visual blurring, flushing, and diarrhea, secondary to excess production of adrenal epinephrine and norepinephrine, as well as vasoactive intestinal polypeptides. Diagnosis is suspected by the increased seric and urinary levels of cathecolamines and metanephrines, and confirmed by the finding of adrenal masses by US, CT, or MRI. ${ }^{123}$ I-meta-iodobenzylguanidine scintigraphy is useful to exclude multifocal disease. In confirmed cases, initial treatment with alpha- and beta-blockers is recommended to control blood pressure and heart rate. Surgical removal of the tumor must be performed under carefully controlled anesthesia with invasive continuous blood pressure and heart rate monitoring, as well as judicious use of nitroprusside and beta-blockers (63). After removal, intensive hydration is necessary to prevent hypotension. Hypertension and other symptoms usually disappear afterward, but control laboratory exams are necessary to identify residual lesions or recurrence.

Although complete removal of the affected adrenal is the standard procedure, partial laparoscopic adrenalectomy, or tumorectomy have gained acceptance for small tumors, particularly in the hereditary syndromes described before. The obvious advantage of partial adrenalectomy is that it may avoid the need of hormonal replacement in cases of bilaterality, although the risk of recurrence in the remnant tissue is also increased $(63,67)$. The laparoscopic approach enables both total and partial adrenalectomy also in children (68).

\section{ADRENOCORTICAL TUMORS}

Primary adrenocortical tumors (ACT) are rare in children, with a worldwide incidence of $0.3 / 1$ million per year before the age of 15 years. In southern Brazil the prevalence is 15 times higher than in other parts of the world, due to TP53 R337H germline mutations in the population of that area $(69,70)$. These tumors occur more often before 5 years of age, with a female predominance. 
Contrary to adults, in children the ACT are generally detected due to the hormonal abnormalities, including virilization in girls, precocious puberty in boys, hypertension, and less often Cushing's syndrome. Increased serum levels of adrenal hormones raise the suspicion of ACT, which are confirmed by CT or MRI, that provide information regarding size and local extension of the tumor, as well as vascular invasion and distant metastases (63).

The characteristics of pediatric ACT vary considerably. Laboratory findings, clinical and hormonal features, as well as tumor size are not able to distinguish adenomas from carcinomas before surgery (71). Small homogeneous masses are usually benign while larger masses with areas of hemorrhage, necrosis, and calcification more often present malignant behavior (63). Although it is difficult to define what is a large tumor in a child, functioning tumors that present heterogeneity, infiltration of vessels or neighboring organs, and a slow wash-out in the image studies are to be removed more expeditiously due to increased risk of malignancy.

After clinical investigation, surgical treatment with radical resection is the treatment of choice, and the hormonal disturbances disappear soon after the removal of the affected gland (64). Laparoscopic adrenalectomy has been performed in children with benign ACT with excellent results (68).

Histologic differentiation of ACT in children is more difficult to define than in adults $(72,73)$. The prognosis is good in young patients with completely removed, non-metastatic, and non-infiltrative lesions. Confirmed cases of carcinoma have an increased risk of recurrence and mortality. COG study ARAR0332 aims to clarify how effective surgery alone is for patients with early stages of ACT, as well as to define the advantages of a more radical surgery with extended lymphadenectomy in selected patients. The efficacy of the combination of mitotane with cisplatin, etoposide, and doxorubicin in advanced stages is also being studied $(64,74,75)$.

\section{REFERENCES}

1. Lowe LH, Isuani BH, Heller RM, Stein SM, Johnson JE, Navarro OM, et al. Pediatric renal masses: Wilms tumor and beyond. Radiographics (2000) 20(6):1585-603. doi:10.1148/radiographics.20.6.g00nv051585

2. Spreafico F, Bellani FF. Wilms' tumor: past, present and (possibly) future. Expert Rev Anticancer Ther (2006) 6(2):249-58. doi:10.1586/14737140.6.2.249

3. Breslow N, Olshan A, Beckwith JB, Green DM. Epidemiology of Wilms tumor. Med Pediatr Oncol (1993) 21(3):172-81. doi:10.1002/mpo.2950210305

4. de Camargo B, de Oliveira Santos M, Rebelo MS, de Souza Reis R, Ferman $\mathrm{S}$, Noronha $\mathrm{CP}$, et al. Cancer incidence among children and adolescents in Brazil: first report of 14 population-based cancer registries. Int J Cancer (2010) 126(3):715-20. doi:10.1002/ijc.24799

5. Dome JS, Fernandez CV, Mullen EA, Kalapurakal JA, Geller JJ, Huff V, et al. Children's Oncology Group's 2013 blueprint for research: renal tumors. Pediatr Blood Cancer (2013) 60:994-1000. doi:10.1002/pbc.24419

6. Geller JI. Genetic stratification of Wilms tumor: is WT1 gene analysis ready for prime time? Cancer (2008) 113(5):893-6. doi:10.1002/cncr.23673

7. Dome JS, Huff V. Wilms tumor overview. In: Pagon RA, Adam MP, Bird TD, Dolan CR, Fong CT, Stephens K editors. GeneReviews ${ }^{\mathrm{TM}}$. Seattle, WA: University of Washington (1993-2013).

8. Perlman EJ. Pediatric renal tumors: practical updates for the pathologist. Pediatr Dev Pathol (2005) 8(3):320-38. doi:10.1007/s10024-005-1156-7

9. Buckley KS. Pediatric genitourinary tumors. Curr Opin Oncol (2012) 24(3):291-6. doi:10.1097/CCO.0b013e32835265c9

10. Ross J. Wilms tumor: updated strategies for evaluation and management. Contemp Urol (2006) 11:18-29.
11. Ko EY, Ritchey ML. Current management of Wilms tumor in children. J Pediatr Urol (2009) 5(1):56-65. doi:10.1016/j.jpurol.2008.08.007

12. Davenport KP, Blanco FC, Sandler AD. Pediatric malignancies: neuroblastoma, Wilm's tumor, hepatoblastoma, rhabdomyosarcoma, and sacroccygeal teratoma. Surg Clin North Am (2012) 92(3):745-67. doi:10.1016/j.suc.2012.03.004

13. Cristófani LM, Duarte RJ, Almeida MT, Odone Filho V, Maksoud JG, Srougi M. Intracaval and intracardiac extension of Wilms tumor. The influence of preoperative chemotherapy on surgical morbidity. Int Braz J Urol (2007) 33(5):683-9. doi:10.1590/S1677-55382007000500010

14. Raval MV, Bilimoria KY, Bentrem DJ, Stewart AK, Winchester DP, Ko CY, et al. Nodal evaluation in Wilms' tumors: analysis of the national cancer data base. Ann Surg (2010) 251(3):559-65. doi:10.1097/SLA.0b013e3181cc95d7

15. Duarte RJ, Dénes FT, Cristofani LM, Odone-Filho V, Srougi M. Further experience with laparoscopic nephrectomy for Wilms' tumour after chemotherapy. BJU Int (2006) 98(1):155-9.

16. Barber TD, Wickiser JE, Wilcox DT, Baker LA. Prechemotherapy laparoscopic nephrectomy for Wilms' tumor. J Pediatr Urol (2009) 5(5):416-9. doi:10.1016/ j.jpurol.2009.01.011

17. Varlet F, Stephan JL, Guye E, Allary R, Berger C, Lopez M. Laparoscopic radical nephrectomy for unilateral renal cell cancer in children. Surg Laparosc Endosc Percutan Tech (2009) 19(2):148-52. doi:10.1097/SLE.0b013e31819f204d

18. Romão RL, Pippi Salle JL, Shuman C, Weksberg R, Figueroa V, Weber B, et al. Nephron sparing surgery for unilateral Wilms tumor in children with predisposing syndromes: single center experience over 10 years. J Urol (2012) 188(4 Suppl):1493-8. doi:10.1016/j.juro.2012.02.034

19. Ferrer FA, Rosen N, Herbst K, Fernandez CV, Khanna G, Dome JS, et al. Image based feasibility of renal sparing surgery for very low risk unilateral Wilms tumors: a report from the Children's Oncology Group. J Urol (2013) 190(5):1846-51. doi:10.1016/j.juro.2013.05.060

20. Davidoff AM, Giel DW, Jones DP, Jenkins JJ, Krasin MJ, Hoffer FA, et al. The feasibility and outcome of nephron-sparing surgery for children with bilateral Wilms tumor. Cancer (2008) 112:2060-70. doi:10.1002/cncr.23406

21. Green DM. The evolution of the treatment for Wilms tumor. J Pediatr Surg (2013) 48(1):14-9. doi:10.1016/j.jpedsurg.2012.10.012

22. Sadak KT, Ritchey ML, Dome JS. Paediatric genitourinary cancers and late effects of the treatment. Nat Rev Urol (2013) 10(1):15-25. doi:10.1038/nrurol.2012.218

23. Termuhlen AM, Tersak JM, Liu Q, Yasui Y, Stovall M, Weathers R, et al. Twenty-five year follow-up of childhood Wilms tumor: a report from the Childhood Cancer Survivor Study. Pediatr Blood Cancer (2011) 57:1210-6. doi:10.1002/pbc. 23090

24. Green DM, Grigoriev YA, Nan B, Takashima JR, Norkool PA, D’Angio GJ, et al. Congestive heart failure after treatment for Wilms' tumor: a report from the National Wilms' Tumor Study group. J Clin Oncol (2001) 19(7): 1926-34.

25. Sandberg AA, Bridge JA. Updates on the cytogenetics and molecular genetics of bone and soft tissue tumors: congenital (infantile) fibrosarcoma and mesoblastic nephroma. Cancer Genet Cytogenet (2002) 132(1):1-13. doi:10.1016/S01654608(02)00592-7

26. Ahmed HU, Arya M, Levitt G, Duffy PG, Mushtaqi I, Sebire N. Part I: primary malignant non-Wilms' renal tumours in children. Lancet Oncol (2007) 8(8):730-7. doi:10.1016/S1470-2045(07)70241-3

27. Eaton KW, Tooke LS, Wainwright LM, Judkins AR, Biegel JA. Spectrum of SMARCB1/INI1 mutations in familial and sporadic rhabdoid tumors. Pediatr Blood Cancer (2011) 56(1):7-15. doi:10.1002/pbc.22831

28. Ahmed HU, Arya M, Levitt G, Duffy PG, Sebire N, Mushtaq I. Part II: treatment of primary malignant non-Wilms' renal tumours in children. Lancet Oncol (2007) 8(9):842-8. doi:10.1016/S1470-2045(07)70241-3

29. Furtwängler R, Gooskens SL, van Tinteren H, de Kraker J, Schleiermacher G, Bergeron $\mathrm{C}$, et al. Clear cell sarcomas of the kidney registered on international society of pediatric oncology (SIOP) 93-01 and SIOP 2001 protocols: a report of the SIOP Renal Tumour Study Group. Eur J Cancer (2013) 49(16):3497-506. doi:10.1016/j.ejca.2013.06.036

30. Selle B, Furtwängler R, Graf N, Kaatsch P, Bruder E, Leuschner I. Populationbased study of renal cell carcinoma in children in Germany, 1980-2005: more frequently localized tumors and underlying disorders compared with adult counterparts. Cancer (2006) 107(12):2906-14. doi:10.1002/cncr.22346

31. Cook A, Lorenzo AJ, Pippi Salle JL, Bakhshi M, Cartwright LM, Bagi D, et al. Pediatric renal cell carcinoma: single institution 25-year case series 
and initial experience with partial nephrectomy. J Urol (2006) 175:1456-60. doi:10.1016/S0022-5347(05)00671-3

32. Leaphart C, Rodeberg D. Pediatric surgical oncology: management of rhabdomyosarcoma. Surg Oncol (2007) 16(3):173-85. doi:10.1016/j.suronc. 2007.07.003

33. Dasgupta R, Rodeberg DA. Update on rhabdomyosarcoma. Semin Pediatr Surg (2012) 21 (1):68-78. doi:10.1053/j.sempedsurg.2011.10.007

34. Loeb DM, Thornton K, Shokek O. Pediatric soft tissue sarcoma. Surg Clin North Am (2008) 88(3):615-27. doi:10.1016/j.suc.2008.03.008

35. Grimsby GM, Ritchey ML. Pediatric urologic oncology. Pediatr Clin North Am (2012) 59(4):947-59. doi:10.1016/j.pcl.2012.05.018

36. Naini S, Etheridge KT, Adam SJ, Qualman SJ, Bentley RC, Counter CM, et al. Defining the cooperative genetic changes that temporally drive alveolar rhabdomyosarcoma. Cancer Res (2008) 68(23):9583-8. doi:10.1158/0008-5472. CAN-07-6178

37. Völker T, Denecke T, Steffen I, Misch D, Schönberger S, Plotkin M, et al. Positron emission tomography for staging of pediatric sarcoma patients: results of a prospective multicenter trial. J Clin Oncol (2007) 25(34):5435-41. doi:10.1200/JCO.2007.12.2473

38. Burnette JO, Klaassen Z, Hatley RM, Neunert CE, Williams H, Donohoe JM. Staging paratesticular rhabdomyosarcoma in the "as low as reasonably achievable" age: the case for PET-CT. Urology (2013) 82:220-4. doi:10.1016/j.urology. 2012.11.051

39. Dang ND, Dang PT, Samuelian J, Paulino AC. Lymph node management in patients with paratesticular rhabdomyosarcoma: a population-based analysis. Cancer (2013) 119(17):3228-33. doi:10.1002/cncr.28198

40. Raney RB, Anderson JR, Barr FG, Donaldson SS, Pappo AS, Qualman SJ, et al. Rhabdomyosarcoma and undifferentiated sarcoma in the first two decades of life: a selective review of intergroup rhabdomyosarcoma study group experience and rationale for Intergroup Rhabdomyosarcoma Study V. J Pediatr Hematol Oncol (2001) 23(4):215-20. doi:10.1097/00043426200105000-00008

41. Crist WM, Anderson JR, Meza JL, Fryer C, Raney RB, Ruyman FB, et al. Intergroup Rhabdomyosarcoma Study-IV: results for patients with nonmetastatic disease. J Clin Oncol (2001) 19(12):3091-102.

42. Wu HY, Snyder HM, Womer RB. Genitourinary rhabdomyosarcoma: which treatment, how much, and when? J Pediatr Urol (2009) 5(6):501-6. doi:10.1016/ j.jpurol.2009.06.011

43. Wiener ES, Anderson JR, Ojimba JI, Lobe TE, Paidas C, Andrassy RJ, et al. Controversies in the management of paratesticular rhabdomyosarcoma: is staging retroperitoneal lymph node dissection necessary for adolescents with resected paratesticular rhabdomyosarcoma? Semin Pediatr Surg (2001) 10(3):146-52. doi:10.1053/spsu.2001.24695

44. Cecchetto G, Carretto E, Bisogno G, Dall'Igna P, Ferrari A, Scarzello G, et al. Complete second look operation and radiotherapy in locally advanced nonalveolar rhabdomyosarcoma in children: a report from the AIEOP soft tissue sarcoma committee. Pediatr Blood Cancer (2008) 51(5):593-7. doi:10.1002/pbc. 21702

45. Rodeberg D, Paidas C. Childhood rhabdomyosarcoma. Semin Pediatr Surg (2006) 15(1):57-62. doi:10.1053/j.sempedsurg.2005.11.009

46. Lerena J, Krauel L, García-Aparicio L, Vallasciani S, Suñol M, Rodó J. Transitional cell carcinoma of the bladder in children and adolescents: six-case series and review of the literature. J Pediatr Urol (2010) 6(5):481-5. doi:10.1016/j. jpurol.2009.11.006

47. Huppmann AR, Pawel BR. Polyps and masses of the pediatric urinary bladder: a 21-year pathology review. Pediatr Dev Pathol (2011) 14(6):438-44. doi:10.2350/11-01-0958-OA.1

48. Bujons A, Caffaratti J, Garat JM, Villavicencio H. Long-term follow-up of transitional cell carcinoma of the bladder in childhood. J Pediatr Urol (2013). doi:10.1016/j.jpurol.2013.08.002

49. Fine SW, Humphrey PA, Dehner LP, Amin MB, Epstein J. Urothelial neoplasms in patients 20 years or younger: a clinico-pathological analysis using the World Health Organization 2004 bladder consensus classification. J Urol (2005) 174:1976-80. doi:10.1097/01.ju.0000176801.16827.82

50. Pohl HG, Shukla AR, Metcalf PD, Cilento BG, Retik AB, Bagli DJ, et al. Prepubertal testis tumors: actual prevalence rate of histological types. J Urol (2004) 172(6):2370-2. doi:10.1097/01.ju.0000144402.13556.74
51. Walsh TJ, Grady RW, Porter MJ, Lin DW, Weiss NS. Incidence of testicular germ cell cancers in U.S. children: SEER program experience 1973 to 2000. Urology (2008) 68(2):402-5. doi:10.1016/j.urology.2006.02.045

52. Rescorla FJ. Pediatric germ cell tumors. Semin Pediatr Surg (2012) 21(1):51-60. doi:10.1053/j.sempedsurg.2011.10.005

53. Kay R. Prepubertal testicular tumor registry. Urol Clin North Am (1993) 20(1):1-5.

54. Metcalfe PD, Farivar-Mohseni J, Farhat W, McLorie G, Khoury A, Bagli DJ. Pediatric testicular tumors: contemporary incidence and efficacy of testicular preserving surgery. J Urol (2003) 170(6):2412-6. doi:10.1097/01.ju.0000097383. 09743.f9

55. Mann JR, Pearson D, Barrett A, Raafat F, Barnes JM, Wallendszus KR. Results of the United Kingdom Children's Cancer Study Group's malignant germ cell tumor studies. Cancer (1989) 63(9):1657-67. doi:10.1002/10970142(19900501)63:9<1657::AID-CNCR2820630902>3.0.CO;2-8

56. Rogers PC, Olson TA, Cullen JW, Billmire DF, Marina N, Rescorla F, et al. Treatment of children and adolescents with stage II testicular and stages I and II ovarian malignant germ cell tumors: a Pediatric Intergroup Study - Pediatric Oncology Group 9048 and Children's Cancer Group 8891. J Clin Oncol (2004) 22(17):3563-9. doi:10.1200/JCO.2004.01.006

57. Cushing B, Giller R, Cullen JW, Marina NM, Lauer SJ, Olson TA, et al. Randomized comparison of combination chemotherapy with etoposide, bleomycin, and either high-dose or standard dose cisplatin in children and adolescents with high-risk malignant germ cell tumors: a pediatric intergroup study - Pediatric Oncology Group 9049 and Children's Cancer Group 8882. J Clin Oncol (2004) 22(13):2691-700. doi:10.1200/JCO.2004.08.015

58. Grady RW, Ross JH, Kay R. Epidemiological features of testicular teratoma in a prepubertal population. J Urol (1997) 158(3):1191-2. doi:10.1097/00005392199709000-00129

59. Rushton G, Belman AB, Sesterhenn I, Patterson K, Mostofi FK. Testicular sparing surgery for prepubertal teratoma of the testis: a clinical and pathological study. J Urol (1990) 144(3):726-30.

60. Ulbright TM. Germ cell tumors of the gonads: a selective review emphasizing problems in differential diagnosis, newly appreciated, and controversial issues. Mod Pathol (2005) 18(Suppl 2):S61-79. doi:10.1038/modpathol.3800310

61. Ross JH. Testicular tumors. In: Gearhart J, Rink R, Mouriquand P editors. Pediatric Urology. Philadelphia: WB Saunders (2001). p. 896-907.

62. Dénes FT, Cocuzza MA, Schneider-Monteiro ED, Silva FA, Costa EM, Mendonça $\mathrm{BBB}$, et al. The laparoscopic management of intersex patients: the preferred approach. BJU Int (2005) 95(6):863-7. doi:10.1111/j.1464-410X.2005. 05417.x

63. Balassy C, Navarro OM, Daneman A. Adrenal masses in children. Radiol Clin North Am (2011) 49(4):711-27. doi:10.1016/j.rcl.2011.05.001

64. Zeiger MA, Thompson GB, Duh QY, Hamrahian AH, Angelos P, Elaraj D, et al. American association of clinical endocrinologists and American association of endocrine surgeons: medical guidelines for the management of adrenal incidentalomas. Endocr Pract (2009) 15(5):450-3. doi:10.4158/EP.15.S1.1

65. Rha SE, Byun JY, Jung SE, Chun HJ, Lee HG, Lee JM. Neurogenic tumors in the abdomen: tumor types and imaging characteristics. Radiographics (2003) 23(1):29-43. doi:10.1148/rg.231025050

66. Park JR, Bagatell R, London WB, Maris JM, Cohn SL, Mattay KM, et al. Children's Oncology Group's 2013 blueprint for research: neuroblastoma. Pediatr Blood Cancer (2013) 60(6):985-93. doi:10.1002/pbc.24433

67. Volkin D, Yerram N, Ahmed F, Lankford D, Baccala A, Gupta GN, et al. Partial adrenalectomy minimizes the need for long-term hormone replacement in pediatric patients with pheochromocytoma and von Hippel-Lindau syndrome. J Pediatr Surg (2012) 47(11):2077-82. doi:10.1016/j.jpedsurg.2012. 07.003

68. Lopes RI, Dénes FT, Bissoli J, Mendonca BB, Srougi M. Laparoscopic adrenalectomy in children. J Pediatr Urol (2012) 8(4):379-85. doi:10.1016/j.jpurol.2011. 07.012

69. Bonfig W, Bittmann I, Bechtold S, Kammer B, Noelle V, Arleth S, et al. Virilising adrenocortical tumours in children. Eur J Pediatr (2003) 162(9):623-8. doi:10.1007/s00431-003-1230-y

70. Marques-Pereira R, Delacerda L, Lacerda HM, Michalkiewicz E, Sandrini F, Sandrini R. Childhood adrenocortical tumours: a review. Hered Cancer Clin Pract (2006) 4(2):81-9. doi:10.1186/1897-4287-4-2-81 
71. Cho MJ, Kim DY, Kim SC, Kim TH, Kim IK. Adrenocortical tumors in children 18 years old and younger. J Korean Surg Soc (2012) 82(4):246-50. doi:10.4174/jkss.2012.82.4.246

72. Lucon AM, Pereira MA, Mendonça BB, Zerbini MC, Saldanha LB, Arap S. Adrenocortical tumors: results of treatment and study of Weiss's score as a prognostic factor. Rev Hosp Clin Fac Med Sao Paulo (2002) 57(6):251-6. doi:10.1590/S0041-87812002000600002

73. Jain M, Kapoor S, Mishra A, Gupta S, Agarwal A. Weiss criteria in large adrenocortical tumors: a validation study. Indian J Pathol Microbiol (2010) 53(2):222-6. doi:10.4103/0377-4929.64325

74. Ribeiro RC, Michalkiewicz EL, Figueiredo BC, DeLacerda L, Sandrini F, Pianovsky MD, et al. Adrenocortical tumors in children. Braz J Med Biol Res (2000) 33(10):1225-34. doi:10.1590/S0100-879X2000001000013

75. Pappo AS, Krailo M, Chen Z, Rodriguez-Galindo C, Reaman G. Infrequent tumor initiative of the Children's Oncology Group: initial lessons learned and their impact on future plans. J Clin Oncol (2010) 28(33):5011-6. doi:10.1200/ JCO.2010.31.2603
Conflict of Interest Statement: The authors declare that the research was conducted in the absence of any commercial or financial relationships that could be construed as a potential conflict of interest.

Received: 03 October 2013; paper pending published: 28 October 2013; accepted: 02 December 2013; published online: 16 December 2013.

Citation: Dénes FT, Duarte RJ, Cristófani LM and Lopes RI (2013) Pediatric genitourinary oncology. Front. Pediatr. 1:48. doi: 10.3389/fped.2013.00048

This article was submitted to Pediatric Urology, a section of the journal Frontiers in Pediatrics.

Copyright (c) 2013 Dénes, Duarte, Cristófani and Lopes. This is an open-access article distributed under the terms of the Creative Commons Attribution License (CC BY).

The use, distribution or reproduction in other forums is permitted, provided the original author(s) orlicensor are credited and that the original publication in this journal is cited, in accordance with accepted academic practice. No use, distribution or reproduction is permitted which does not comply with these terms. 